NIKHEF/99-033

\title{
NLO Calculations for Charm Production in DIS
}

\author{
Eric Laenen $\dagger$ \\ $\dagger$ NIKHEF Theory Group, Kruislaan 409, 1009 DB, Amsterdam, The Netherlands
}

\begin{abstract}
I present a short overview of the NLO QCD calculations available for deep-inelastic production of heavy quarks.
\end{abstract}

\section{Introduction}

Charm quarks produced in deep-inelastic scattering (DIS) have been identified in sizable numbers now by the H1 [1] and ZEUS [2] collaborations at HERA, and considerably more charm (and bottom) data are anticipated. At the theoretical level the reaction has already been studied extensively. In the framework where the heavy quark is not treated as a parton, leading order (LO) [3, 4] and next-to-leading order (NLO) [5, 6] calculations of the inclusive structure functions exist. Moreover, LO (AROMA, RAPGAP) [7, 8] and NLO (HVQDIS) [9, 10, 11] Monte-Carlo programs, allowing a much larger class of observables to be compared with data, have been constructed in recent years. Overall, the NLO QCD description agrees quite well with the HERA data. Here I shall give a very brief overview of these NLO calculations.

Charm quarks are produced in DIS via the reaction

$$
e^{ \pm}\left(p_{e}\right)+P(p) \rightarrow e^{ \pm}\left(p_{e}-q\right)+X[Q, \bar{Q}],
$$

where $P(p)$ is a proton with momentum $p, Q$ is a heavy quark with momentum $p_{1}$ $\left(p_{1}^{2}=m^{2}\right)$ and $X$ is any hadronic state allowed, containing the heavy quark-antiquark pair. Its differential cross section may be expressed in general as

$\frac{d^{2+n} \sigma}{d x d Q^{2} \prod_{i} d V_{i}}=\frac{2 \pi \alpha^{2}}{x Q^{4}}\left[\left(1+(1-y)^{2}\right) \frac{d^{n} F_{2}}{\prod_{i} d V_{i}}\left(x, Q^{2}, m^{2}, V_{i}\right)-y^{2} \frac{d^{n} F_{L}}{\prod_{i} d V_{i}}\left(x, Q^{2}, m^{2}\right)\right]$,

where

$$
Q^{2}=-q^{2}, \quad x=\frac{Q^{2}}{2 p \cdot q}, \quad y=\frac{p \cdot q}{p \cdot p_{e}} .
$$

The $V_{i}$ stand for kinematic variables related to the heavy quarks. Examples are the transverse momentum of the heavy quark, the rapidity difference between the heavy quarks, etc. 


\section{Inclusive and single-charm inclusive production}

The least difficult cross section to measure is the heavy quark inclusive cross section, expressed via (2) in the inclusive structure functions $F_{2}$ and $F_{L}$. These were calculated to NLO in [5]. The results are parametrized as

$$
\begin{aligned}
F_{k}\left(x, Q^{2}, m^{2}\right) & =\frac{Q^{2} \alpha_{s}}{4 \pi^{2} m^{2}} \int_{x}^{z_{\max }} \frac{d z}{z}\left[e_{H}^{2} f_{g}\left(\frac{x}{z}, \mu^{2}\right) c_{k, g}^{(0)}\right] \\
& +\frac{Q^{2} \alpha_{s}^{2}}{\pi m^{2}} \int_{x}^{z_{\max }} \frac{d z}{z}\left[e_{H}^{2} f_{g}\left(\frac{x}{z}, \mu^{2}\right)\left(c_{k, g}^{(1)}+\bar{c}_{k, g}^{(1)} \ln \frac{\mu^{2}}{m^{2}}\right)\right. \\
& \left.+\sum_{i=q, \bar{q}}\left[e_{H}^{2} f_{i}\left(\frac{x}{z}, \mu^{2}\right)\left(c_{k, i}^{(1)}+\bar{c}_{k, i}^{(1)} \ln \frac{\mu^{2}}{m^{2}}\right)+e_{L, i}^{2} f_{i}\left(\frac{x}{z}, \mu^{2}\right) d_{k, i}^{(1)}\right]\right],
\end{aligned}
$$

where $k=2, L$ and the upper boundary on the integration is given by $z_{\max }=$ $Q^{2} /\left(Q^{2}+4 m^{2}\right)$. The parton densities $f_{i}\left(x, \mu^{2}\right),(i=g, q, \bar{q})$ are explicitly identified. The scale $\mu$ is the mass factorization and renormalization scale. The $\overline{\mathrm{MS}}$ coefficient functions $c_{k, j}^{(l)}(\eta, \xi), \bar{c}_{k, j}^{(l)}(\eta, \xi),(j=g, q, \bar{q} ; l=0,1)$ and $d_{k, i}^{(l)}(\eta, \xi),(i=q, \bar{q} ; l=0,1)$ depend on the scaling variables $\eta=\left(s-4 m^{2}\right) / 4 m^{2}$ and $\xi=Q^{2} / m^{2}$, with $s$ the square of the c.m. energy of the virtual photon-parton subprocess. This implies that in (4) $z=Q^{2} /\left(Q^{2}+s\right)$.

In (4) we distinguish the coefficient functions with by their origin. The $c_{k, i}^{(l)}(\eta, \xi), \bar{c}_{k, i}^{(l)}(\eta, \xi)$ originate from those partonic subprocesses where the virtual photon is coupled to the heavy quark, whereas the $d_{k, i}^{(l)}(\eta, \xi)$ correspond to the subprocess where the virtual photon interacts with the light quark. Thus the former are multiplied by the charge squared of the heavy quark $e_{H}^{2}$, and the latter by the charge squared of the light

quark $e_{L}^{2}$ respectively (both in units of $e$ ). Only the terms proportial to $e_{H}^{2}$ contain the gluon density.

To obtain numerical results for the inclusive cross section, it is better to use instead of the original, rather long expressions [5], the much faster parametrized form [6]. The lowest order term contains only the gluon density. Light quark densities only come in at next order, contributing only about $5 \%$. This is the reason $F_{2}\left(x, Q^{2}, m^{2}\right)$ is used in global analyses to constrain the gluon density. Besides the gluon density, the main source of theoretical uncertainty in $F_{2}\left(x, Q^{2}, m^{2}\right)$ is the value of the charm quark (pole) mass, rather than the scale $\mu$.

The calculation that lead to (4) also yielded the single heavy quark differential cross section, with $V_{1}=p_{T}^{Q}$ and $V_{2}=y^{Q}[12]$. These distributions are best generated using the HVQDIS program, described in the next section.

NNLO estimates based on soft gluon resummation are given in Ref. [13], for inclusive and single heavy quark inclusive cross section.

\section{Fully differential charm production}

A NLO calculation also exists for the fully differential cross section in (2) [14]. Maintaining full differentiality required a complete recalculation of the matrix elements, 
carefully eliminating intermediate divergences via the so-called subtraction method. The results are encoded in the program HVQDIS [10] $\ddagger$. The program can compute, to NLO, experimentally visible cross sections, which are in principle better for comparison with theory than fully inclusive ones. It returns parton kinematic configurations and their

corresponding weights, accurate to $\mathcal{O}\left(\alpha \alpha_{s}^{2}\right)$. The user is free to histogram any set of infrared-safe observables and apply cuts, all in a single histogramming subroutine.

Additionally, one may study heavy hadrons using the Peterson et al. fragmentation model. Detailed physics results from this program are given in [11].

HVQDIS has been used extensively in experimental analyses. As is shown elsewhere [16] in these proceedings, it reproduces the data very well indeed, except for $D^{*}$ 's at low $p_{T}$ and large pseudorapidity, where there are more events than HVQDIS would predict. This is possibly due to remnant beam drag effects distorting the pseudorapidity spectrum to larger values. For the case of charm photoproduction this was investigated in [17].

A more extensive overview of the NLO calculations and the phenomenology of DIS charm production can be found in [18].

\section{References}

[1] H1, C. Adloff et al., Z. Phys. C72, 593 (1996), hep-ex/9607012.

[2] ZEUS, J. Breitweg et al., Phys. Lett. B407, 402 (1997), hep-ex/9706009.

[3] E. Witten, Nucl. Phys. B104, 445 (1976).

[4] M. Gluck and E. Reya, Phys. Lett. 83B, 98 (1979).

[5] E. Laenen, S. Riemersma, J. Smith, and W. L. van Neerven, Nucl. Phys. B392, 162 (1993).

[6] S. Riemersma, J. Smith, and W. L. van Neerven, Phys. Lett. B347, 143 (1995), hep-ph/9411431.

[7] G. Ingelman, J. Rathsman, and G. A. Schuler, Comput. Phys. Commun. 101, 135 (1997), hep$\mathrm{ph} / 9605285$.

[8] H. Jung, http://www-h1.desy.de/ jung/rapgap/rapgap.html.

[9] B. W. Harris and J. Smith, Nucl. Phys. B452, 109 (1995), hep-ph/9503484.

[10] B. W. Harris, (1996), hep-ph/9608379, in Minneapolis Meeting of the Division of Particles and Fields of the American Physical Society, Minneapolis, 1996, edited by J.K. Nelson and K. Heller (World Scientific, Singapore, 1996), p. 1019.

[11] B. W. Harris and J. Smith, Phys. Rev. D57, 2806 (1998), hep-ph/9706334.

[12] E. Laenen, S. Riemersma, J. Smith, and W. L. van Neerven, Nucl. Phys. B392, 229 (1993).

[13] E. Laenen and S.-O. Moch, Phys. Rev. D59, 034027 (1999), hep-ph/9809550.

[14] J. Smith and B. W. Harris, Nucl. Phys. Proc. Suppl. 51C, 188 (1996), hep-ph/9605358.

[15] B. W. Harris, E. Laenen, S. Moch, and J. Smith, (1998), hep-ph/9905365.

[16] M. R. Sutton, these proceedings.

[17] E. Norrbin and T. Sjostrand, (1999), hep-ph/9905493.

[18] B. W. Harris, (1999), hep-ph/9909310.

‡ See [15] for a description of the most recent changes. 